This article may be downloaded for personal use only. Any other use requires prior permission of the author and AIP Publishing. This article appeared in Appl. Phys. Lett. 114, 103504 (2019) and may be found at https:// doi.org/10.1063/1.5087058.

\title{
Quasi-phase-matched second harmonic generation of UV light using AIN waveguides
}

F

Cite as: Appl. Phys. Lett. 114, 103504 (2019); https://doi.org/10.1063/1.5087058

Submitted: 27 December 2018 . Accepted: 30 January 2019 . Published Online: 14 March 2019

Dorian Alden (D), Tinkara Troha (D), Ronny Kirste, Seiji Mita, Qiang Guo, Axel Hoffmann, Marko Zgonik (D), Ramón Collazo, and Zlatko Sitar (iD)

\section{COLLECTIONS}

F This paper was selected as Featured
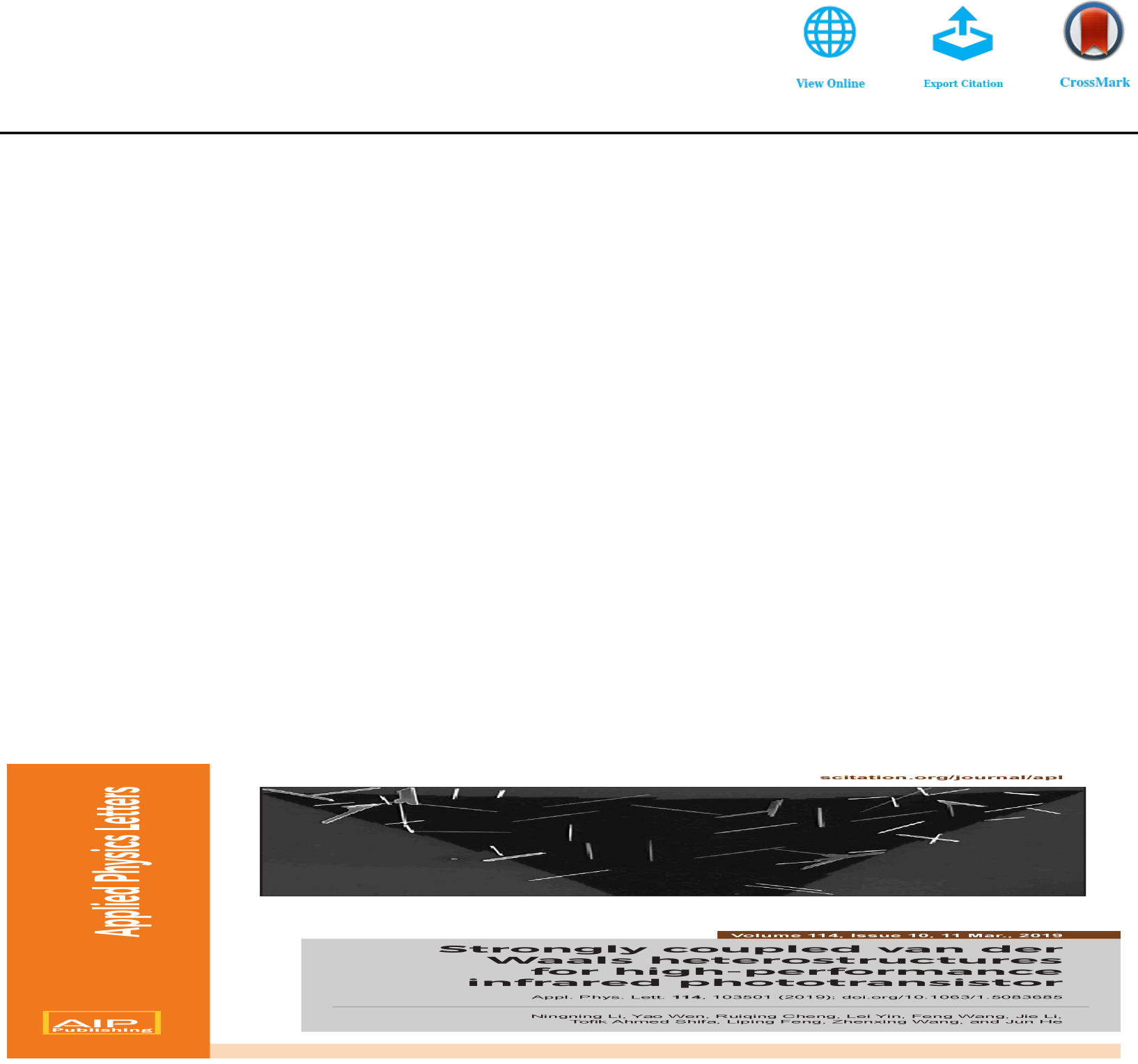


\title{
Quasi-phase-matched second harmonic generation of UV light using AIN waveguides $\circledast 9$
}

\author{
Cite as: Appl. Phys. Lett. 114, 103504 (2019); doi: 10.1063/1.5087058 \\ Submitted: 27 December 2018 - Accepted: 30 January 2019 . \\ Published Online: 14 March 2019
}

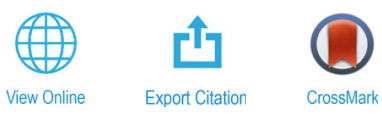

\author{
Dorian Alden, ${ }^{1,2}$ (D) Tinkara Troha, ${ }^{3}$ (D) Ronny Kirste, ${ }^{1,4, a)}$ Seiji Mita, ${ }^{1,4}$ Qiang Guo, Axel Hoffmann, ${ }^{2}$ Marko Zgonik, ${ }^{3,5}$ iD \\ Ramón Collazo, and Zlatko Sitar ${ }^{1,4}$
}

\author{
AFFILIATIONS \\ 'Department of Materials Science and Engineering, North Carolina State University, Raleigh, North Carolina 27695, USA \\ ${ }^{2}$ Institut für Festkörperphysik, Technische Universität Berlin, Hardenbergstr. 36, 10623 Berlin, Germany \\ ${ }^{3}$ Faculty of Mathematics and Physics, University of Ljubljana, Jadranska 19, 1000 Ljubljana, Slovenia \\ ${ }^{4}$ Adroit Materials, Inc., 2054 Kildaire Farm Rd., Suite 205, Cary, North Carolina 27518, USA \\ ${ }^{5}$ Complex Matter Department, J. Stefan Institute, Jamova 39, SI-1000 Ljubljana, Slovenia
}

a) Author to whom correspondence should be addressed: rkirste@ncsu.edu

\begin{abstract}
As an alternative to electrically injected diodes, UV light emission can be obtained via second harmonic generation (SHG). In weakly birefringent materials such as aluminum nitride (AlN), the phase matching of the driving and second harmonic waves can be achieved by the quasi-phase-matching (QPM) technique, where the polarity of the material is periodically changed commensurate with the coherence wavelength. QPM also allows the use of the highest nonlinear susceptibility, and therefore, higher conversion efficiencies are possible. In this work, the QPM SHG of UV light in AlN lateral polar structure-based waveguides is demonstrated. The peak intensity of the frequency doubled laser light was measured at $344 \mathrm{~nm}$ and $472 \mathrm{~nm}$ wavelengths, in agreement with dispersion-based theoretical predictions. These results confirm the potential of III-nitride-based lateral polar structures for quasi-phase-matched nonlinear optics and for frequency doubling media for UV light generation.
\end{abstract}

Published under license by AIP Publishing. https://doi.org/10.1063/1.5087058

Compact UV laser sources are envisioned for applications in disinfection, sensing, quantum cryptography, and communications. ${ }^{1-3}$ Current UV laser sources are either gas-based (e.g., ArF, $\mathrm{KrF}$, and Ar-ion), or they exploit higher harmonics generated from infrared lasers (Nd:YAG and Ti:sapphire). For the latter, an efficient crystal for second (or higher) harmonic generation is needed. AlN is an excellent candidate for the generation of UV light via second harmonic generation (SHG) due to its large second order nonlinear susceptibility coefficient along the c-axis, with values between 4 and $7 \mathrm{pm} / \mathrm{V}$ in the spectral region interesting for UV generation, ${ }^{4}$ which is superior to that of currently employed nonlinear crystals, such as $\beta-\mathrm{BaB}_{2} \mathrm{O}_{4}(1.84 \mathrm{pm} / \mathrm{V}){ }^{5}$ In addition, AlN has a high thermal conductivity $(\sim 370 \mathrm{~W} / \mathrm{mK})^{6,7}$ and a wide transparency window $(205 \mathrm{~nm}-15 \mu \mathrm{m}),{ }^{8}$ resulting in a high power damage threshold and wide spectral tunability. Since birefringent phase matching is not accessible in AlN, the SHG of laser light utilizing quasi-phase-matching (QPM) seems to be the most promising alternative. QPM can be achieved by periodically changing the sign of the susceptibility coefficient in the host material and can be very efficient since it allows access to the largest nonlinear susceptibility coefficient of the material. ${ }^{9-11}$ A prominent example is periodically poled lithium niobate (PPLN). ${ }^{11,12}$ In III-nitrides such as GaN or AlN, QPM can be achieved by periodically inverting the direction of the c-axis or the polarity of the material. ${ }^{13-17}$ Depending on the targeted wavelength, this change in polarity must be achieved on the micrometer or even on the nanometer length scale. This precise control of polarity and surface roughness is challenging and so far has limited the development of AlN based QPM structures. ${ }^{18}$

In this article, we report on the demonstration of the QPM SHG of UV light at $344 \mathrm{~nm}$, using a $0.5 \mu \mathrm{m}$ thick AlN lateral polar structure-based waveguide with a periodicity of $10 \mu \mathrm{m}$. The control of the surface and interfaces of the Al- and N-polar domains is the biggest challenge to reduce scattering and increase the conversion efficiency. The observed SHG wavelength is found to be in excellent agreement with theoretical predictions based on the QPM approach and expected waveguide propagation modes. These results highlight the potential of III-nitride-based 
waveguide structures for QPM applications in the UV wavelength region.

AlN layers were grown on sapphire using metalorganic chemical vapor phase deposition (MOCVD). The control of the polarity on the micrometer length scale and the growth of lateral polar structures (LPS) was achieved using patterned AlN buffer layers. Details on the AlN LPS growth and fabrication are discussed elsewhere. ${ }^{18,19}$ The main challenge in the growth of LPS is to minimize surface scattering to control optical loss. Surface scattering arises primarily due to two reasons: different growth rates and resulting heights between the $\mathrm{N}$ - and Al-polar domains and a relatively rough $\mathrm{N}$-polar AlN surface. . $^{1,20,21}$ Therefore, a thorough control of the surface morphology of both polar domains is crucial to achieve low loss LPS waveguides. Here, a supersaturation scheme was followed, which led to a high temperature growth of the AlN LPS to enable the growth of smooth $\mathrm{N}$-polar domains and to equalize the growth rate of both types of domains. ${ }^{18,22}$ No additional effort was made to achieve smooth AlN LPS. The $10 \mu$ m periodic AlN LPS used for the fabrication of waveguides was grown at $1500{ }^{\circ} \mathrm{C}$ and had typically an RMS roughness of $\sim 10 \mathrm{~nm}$ as measured over $90 \times 90 \mu \mathrm{m}^{2}$. This value is dominated by the $\mathrm{N}$-polar region since the RMS value of the Al-polar domain is around $1 \mathrm{~nm}$. Subsequently, a photoresist, in combination with reactive ion etching, was used to fabricate various waveguides with a width in the range of $4-10 \mu \mathrm{m}$ and periodically inverted domains. Figure 1 shows a representative section of a fabricated $500 \mathrm{~nm}$ thick and $4 \mu \mathrm{m}$ wide stripe waveguide.

Second harmonic generation measurements were conducted in an end fire-coupling geometry (Fig. 2) using a Ti:sapphire femtosecond pulsed laser system with a tunable wavelength range between $600 \mathrm{~nm}$ and $1000 \mathrm{~nm}$. Laser pulses had a pulse duration of $40 \mathrm{fs}$, a spectral width of $30 \mathrm{~nm}$ (FWHM), and the pulse energy ranging from 1 to $100 \mu \mathrm{J}$, depending on the selected wavelength. The fundamental laser was coupled to the waveguide facet using a lens with a focal length of $10 \mathrm{~mm}$. The polarization of the laser was set to allow the propagation of transverse magnetic (TM) modes through the waveguide. At the opposing waveguide facet, the generated second harmonic ( $\mathrm{SH})$ signal was out-coupled and separated from the remaining fundamental signal. The SH signal was guided to a spectrometer
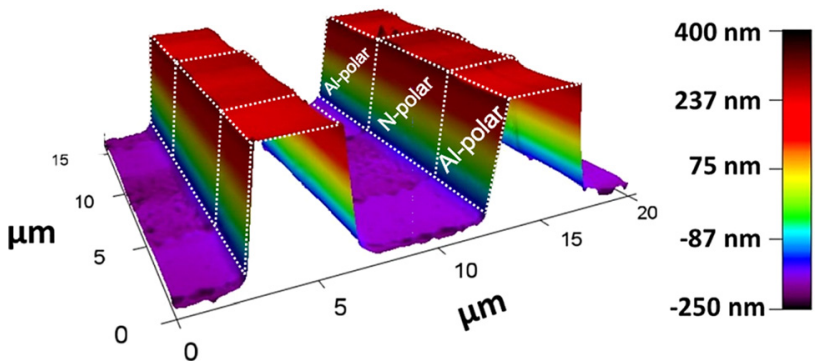

FIG. 1. 3D AFM topograph of two neighboring periodically poled AIN waveguides. The periodicity of the LPS was $10 \mu \mathrm{m}$. Note the different vertical and lateral length scales: the waveguides are $500 \mathrm{~nm}$ high and $4 \mu \mathrm{m}$ wide. White dotted lines indicate the domain boundaries.

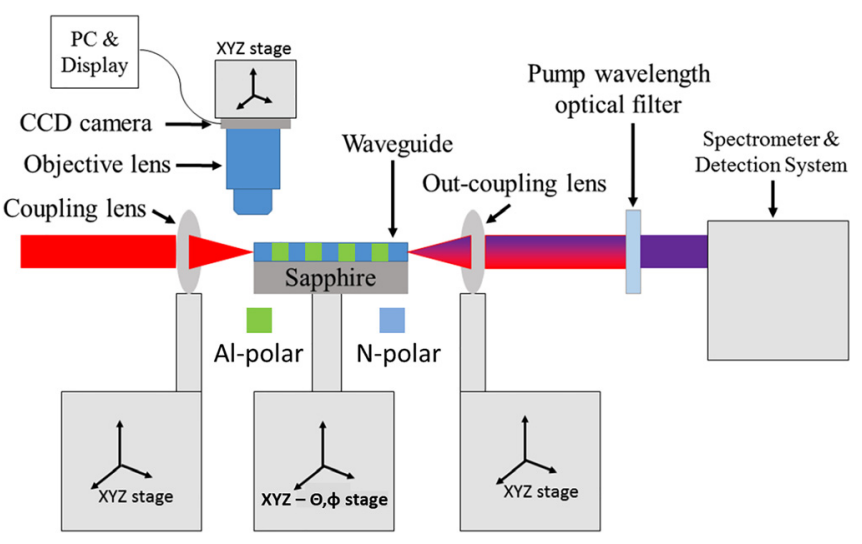

FIG. 2. Schematic of the experimental setup used for second harmonic generation in AIN-based lateral polar structures.

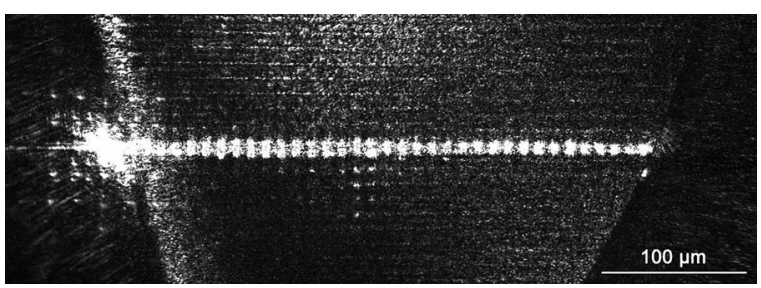

FIG. 3. Top view of the scattering intensity of the laser light propagating through an AIN LPS-based waveguide with a domain periodicity of $10 \mu \mathrm{m}$. Scattering in the Npolar domains is significantly higher (bright regions) than in the Ga-polar domains (dark regions).

and detected using a photon counting camera operated in a gated mode synchronized with the femtosecond laser system.

The waveguides were tested for light transmission using the setup depicted in Fig. 2 and a HeNe laser as an excitation source with an excitation power of around $95 \mu \mathrm{W}$. Figure 3 shows the top view of a waveguide depicting the scattering light

$$
\begin{array}{r}
\text { QPM condition } \\
\boldsymbol{n}_{\omega, \text { eff }}=n_{2 \omega, \text { eff }}-N \frac{\lambda_{0}}{2 \Lambda}
\end{array}
$$

$540 \mathrm{~nm}$ thick waveguide

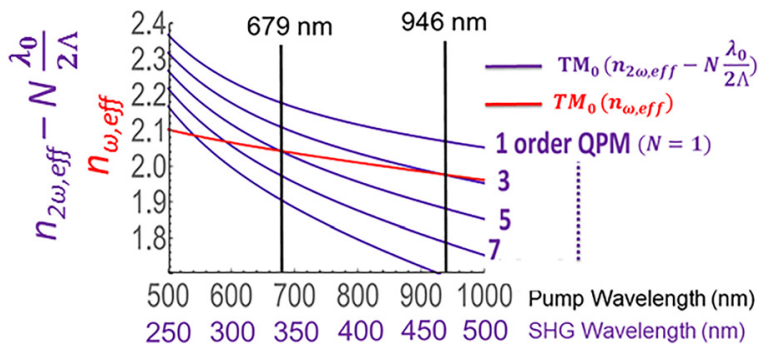

FIG. 4. Calculated dispersion relation for the Oth $\mathrm{TM}_{0}$ waveguide mode for the fundamental wave (red) and second harmonic waves (purple) for several orders $(N=1,3,5$, and 7$)$ of quasi-phase-matching conditions. 

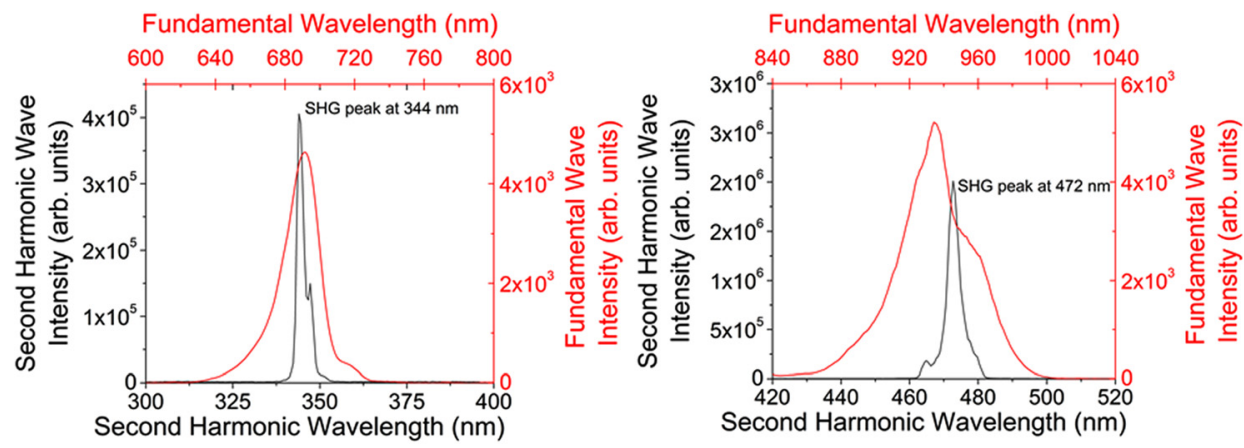

FIG. 5. Measured spectral intensity of the second harmonic generated UV laser light via a quasi-phase-matching in AIN lateral polar structure waveguides with a periodicity of $10 \mu \mathrm{m}$ at $344 \mathrm{~nm}$ (left) and $472 \mathrm{~nm}$ (right).

intensity of the laser light traveling through a $400 \mu \mathrm{m}$ long AlN LPS-based waveguide with a domain periodicity of $10 \mu \mathrm{m}$. Strong scattering is observed at the in-coupling facet; appreciable scattering was also observed at the out-coupling facet. In addition, scattering is also observed from the waveguide region itself. A closer look at the scattering intensity profile along the waveguide reveals a periodic pattern. The periodicity of the scattering intensity is found to match the AlN LPS periodicity of $10 \mu \mathrm{m}$. While light was guided through the whole waveguide, this scattering is still a loss mechanism which reduces the efficiency of QPM second harmonic generation. Based on the surface roughness measured in Fig. 1, the average scattering loss at the waveguide surface was calculated to be $\sim 10 \mathrm{~cm}^{-1}$. This once again highlights the importance of achieving a smooth waveguide surface to minimize scattering losses.

Quasi-phase-matching in AlN based LPS is achieved by changing the sign of the susceptibility coefficient $d_{33}$, after odd multiples of the coherence length $l_{\text {coh }}$ according to

$$
\Lambda=2 \mathrm{~N} \times l_{\mathrm{coh}}=2 \mathrm{~N} \times\left(\frac{\lambda_{0}}{4\left(n_{2 \omega, e f f}-n_{\omega, e f f}\right)}\right),
$$

where $\Lambda$ is the periodicity of the LPS structure, $\lambda_{0}$ is the pump wavelength, $\mathrm{N}$ is the phase matching order, and $n_{i}$ are the effective refractive indices at the pump and the second harmonic wavelength. ${ }^{9,10}$ Equation (1) can be conveniently expressed as

$$
n_{\omega, e f f}=n_{2 \omega, e f f}-\mathrm{N} \frac{\lambda_{0}}{2 \Lambda}
$$

to allow graphical representation of solutions. Figure 4 shows the dispersion relations for the effective refractive indices of the fundamental (red) and second harmonic waves (purple) as calculated for the waveguide dimensions extracted from the AFM image in Fig. 1. The right side of Eq. (2) is plotted for the odd values of $N$ to illustrate the conditions for which QPM is expected. The intersection between the red and purple lines corresponds to the predicted wavelength for SHG. Based on Fig. 4, it is found that for $540 \mathrm{~nm}$ thick AlN LPS-based waveguides with a domain periodicity of $10 \mu \mathrm{m}$, one should expect for the $\mathrm{TM}_{0}$ modes $3 \mathrm{rd}$ and 5th order QPM SHG at $340 \mathrm{~nm}$ and $473 \mathrm{~nm}$ for pump wavelengths of $679 \mathrm{~nm}$ and $946 \mathrm{~nm}$, respectively.

Phase matching for AlN LPS with a periodicity of $10 \mu \mathrm{m}$ and a length of $400 \mu \mathrm{m}$ was investigated. Therefore, pump light
(Ti:sapphire) was coupled to the waveguides, and the outcoupled light was monitored. The SH response was monitored, and the position of the sample and in- and out-coupling lenses were adjusted until the maximum intensity of the $\mathrm{SH}$ light was achieved. The corresponding tuning curves were recorded and analyzed. Figure 5 displays the spectra of the pump laser at $690 \mathrm{~nm}$ and $940 \mathrm{~nm}$ (Ti:sapphire laser) and the corresponding second harmonic responses at $344 \mathrm{~nm}$ and $472 \mathrm{~nm}$, respectively. These spectra correspond to the highest SH intensity in the tuning curves. The observed SHG wavelengths are in excellent agreement with the theoretical calculations shown in Fig. 4. Theory predicts the 3rd and 5th order QPM SHG at $340 \mathrm{~nm}$ and $473 \mathrm{~nm}$, respectively, where the $\mathrm{TM}_{0}$ modes of the fundamental and second harmonic waves are phase-matched. The minor difference between the theoretical and experimental values can be explained by variations in the waveguide thickness due to the surface roughness or small errors in the refractive index. ${ }^{23}$ While the efficiency of the SHG via quasi-phase-matching can be high, here, as evidenced in Fig. 3, scattering losses and coupling losses are not negligible and the conversion efficiency is expected to be $<1 \%$. This is explained by the remaining surface roughness of the LPS. ${ }^{24}$ Although all these challenges need to be addressed in future research, there seem to be no fundamental obstacles to high efficiency QPM second harmonic generation in AlN-based LPS.

In summary, the feasibility of quasi-phase-matched UV second harmonic generation in AlN LPS-based waveguides for QPM was demonstrated. Second harmonic light generation at $344 \mathrm{~nm}$ and $472 \mathrm{~nm}$ was observed using a tunable Ti:sapphire as a pump source at $690 \mathrm{~nm}$ and $940 \mathrm{~nm}$, respectively. The observed SH wavelengths were in excellent agreement with theoretical predictions. While the observed efficiency was low, further improvements related to the surface roughness and light coupling are expected to have a significant impact on the conversion efficiency.

D. Alden would like to acknowledge CONACYT-Mexico for their financial support. Partial financial support from NSF (DMR-1108071, DMR-1312582, ECCS-1508854, and DMR1508191) and ARO (W911NF-15-2-0068 and W911NF-14-C-0008) is acknowledged. This work was also financially supported by the Slovenian Research Agency research core funding (P10192) and project (N1-0021). 


\section{REFERENCES}

${ }^{1}$ R. Kirste, N. Rohrbaugh, I. Bryan, Z. Zryan, R. Collazo, and A. Ivanisevic, Annu. Rev. Anal. Chem. 8, 149 (2015).

${ }^{2}$ M. Kneissl, "A brief review of III-Nitride UV emitter technologies and their applications," in III-Nitride Ultraviolet Emitters: Technology and Applications, edited by M. Kneissl and J. Rass (Springer International Publishing, Cham, 2016), pp. 1-25.

${ }^{3}$ R. Kirste, Q. Guo, J. H. Dycus, A. Franke, S. Mita, B. Sarkar, P. Reddy, J. M. LeBeau, R. Collazo, and Z. Sitar, Appl. Phys. Express 11, 082101 (2018).

${ }^{4}$ Y. Fujii, S. Yoshida, S. Misawa, S. Maekawa, and T. Sakudo, Appl. Phys. Lett, 31, 815-816 (1977).

${ }^{5}$ T. Sasaki, Y. Mori, M. Yoshimura, Y. K. Yap, and T. Kamimura, Mater, Sci. Eng.: R: Rep. 30, 1-54 (2000).

${ }^{6}$ G. A. Slack, R. A. Tanzilli, R. O. Pohl, and J. W. Vandersande, J. Phys. Chem. Solids 48, 641-647 (1987).

${ }^{7}$ R. Rounds, B. Sarkar, A. Klump, C. Hartmann, T. Nagashima, R. Kirste, A. Franke, M. Bickermann, Y. Kumagai, Z. Sitar, and R. Collazo, Appl. Phys. Express 11, 071001 (2018).

${ }^{8}$ W. Yim, J. Appl. Phys. 44, 292 (1973).

9J. A. Armstrong, N. Bloembergen, J. Ducuing, and P. S. Pershan, Phys. Rev. 127, 1918-1939 (1962).

${ }^{10}$ M. M. Fejer, G. Magel, D. H. Jundt, and R. L. Byer, IEEE J. Quantum Electron. 28, 2631-2654 (1992).

${ }^{11}$ S. Sinha, D. S. Hum, K. Urbanek, Y.-W. Lee, M. J. F. Digonnet, M. M. Fejer, and R. L. Byer, J. Lightwave Technol. 26, 3866-3871 (2008).

${ }^{12}$ K. Mizuuchi, A. Morikawa, T. Sugita, and K. Yamamoto, Jpn. J. Appl. Phys. Part 2 42, L90 (2003).

${ }^{13}$ M. Stutzmann, O. Ambacher, M. Eickhoff, U. Karrer, A. Lima Pimenta, R. Neuberger, J. Schalwig, R. Dimitrov, P. Schuck, and R. Grober, Phys. Status Solidi B 228, 505-512 (2001).
${ }^{14}$ J. Hite, M. Twigg, M. Mastro, J. Freitas, J. Meyer, I. Vurgaftman, S. óConnor, N. Condon, F. Kub, S. Bowman, and C. Eddy, Opt. Mater. Express 2, 1203-1208 (2012).

${ }^{15}$ J. Hite, N. Y. Garces, R. Goswami, M. A. Mastro, F. J. Kub, and C. R. Eddy, Jr., Appl. Phys. Express 7, 025502 (2014).

${ }^{16}$ C. G. Brown, S. R. Bowman, J. K. Hite, J. A. Freitas, F. J. Kub, C. R. Eddy, I. Vurgaftman, J. R. Meyer, J. H. Leach, and K. Udwary, Frequency Conversion in Free-Standing Periodically Oriented Gallium Nitride, (SPIE LASE, 2016).

${ }^{17}$ A. Chowdhury, H. M. Ng, M. Bhardwaj, and N. G. Weimann, Appl. Phys. Lett. 83, 1077-1079 (2003).

${ }^{18}$ D. Alden, W. Guo, R. Kirste, F. Kaess, I. Bryan, T. Troha, A. Bagal, P. Reddy, L. H. Hernandez-Balderrama, A. Franke, S. Mita, C.-H. Chang, A. Hoffmann, M. Zgonik, R. Collazo, and Z. Sitar, Appl. Phys. Lett. 108, 261106 (2016).

${ }^{19}$ R. Kirste, S. Mita, M. P. Hoffmann, L. Hussey, W. Guo, I. Bryan, Z. Bryan, J. Tweedie, M. Gerhold, A. Hoffmann, R. Collazo, and Z. Sitar, Phys. Status Solidi C 11, 261-264 (2014).

${ }^{20}$ R. Kirste, R. Collazo, G. Callsen, M. R. Wagner, T. Kure, J. S. Reparaz, S. Mita, J. Xie, A. Rice, J. Tweedie, Z. Sitar, and A. Hoffmann, J. Appl. Phys. 110, 093503-9 (2011).

${ }^{21}$ R. Kirste, S. Mita, L. Hussey, M. P. Hoffmann, W. Guo, I. Bryan, Z. Bryan, J. Tweedie, J. Xie, M. Gerhold, R. Collazo, and Z. Sitar, Appl. Phys. Lett. 102, 181913-4 (2013).

${ }^{22}$ D. Alden, "Periodic lateral polar structure-based waveguides in IIInitrides for quasi-phase matched second harmonic generation," Dissertation (Technische Universität Berlin, 2017).

${ }^{23}$ M. Rigler, M. Zgonik, M. P. Hoffmann, R. Kirste, M. Bobea, R. Collazo, Z. Sitar, S. Mita, and M. Gerhold, Appl. Phys. Lett. 102, 221106-5 (2013).

${ }^{24}$ D. Taverner, P. Britton, P. G. R. Smith, D. J. Richardson, G. W. Ross, and D. C. Hanna, Opt. Lett. 23, 162-164 (1998). 